\title{
Full Body Interaction with Virtual Characters in an Interactive Storytelling Scenario
}

\author{
Felix Kistler, Birgit Endrass, and Elisabeth André \\ Human Centered Multimedia, Augsburg University, \\ Universitätsstr. 6a, 86159 Augsburg, Germany \\ $\{$ kistler, endrass, andre $\} @ \mathrm{hcm}-\mathrm{lab} . \mathrm{de}$
}

\begin{abstract}
This paper presents a full body interaction approach developed for Traveller, an intercultural training game for young adults based on an interactive storytelling scenario. Traveller involves virtual characters interacting with the users on a large display screen. The users interact with a Kinect, performing full body gestures and controlling a freehand swipe menu to trigger navigation and dialogue actions in the game. A first evaluation proved the recognition capabilities of our system and the comparison with a mouse interface in terms of usability and user experience showed higher positive affect with the Kinect, but a tendency for higher usability and flow with the mouse.
\end{abstract}

\section{Introduction}

To support experiential learning in simulation environments, technologies are required that allow for intuitive and natural forms of interaction. Frequently, such simulation environments are inhabited by virtual characters. To provide basic interaction with the characters, users need to be able to move towards the characters (navigation) and communicate with them (dialogue). Traditionally, navigation and dialogue have been controlled with keyboard, mouse or joystick input often accompanied by a graphical interface [9]. However, this does not allow for human-like interaction styles and may affect the user experience. Better options are speech $[3,5]$ or gesture input $[6,1]$ that emulate human-human conversation in a more direct manner. While there is empirical evidence that bodily interaction contributes to a greater sense of presence, some studies also revealed usability issues that negatively affected user experience. For example, Dow and colleagues [3] revealed that interaction in Augmented Reality contributed to an enhanced sense of presence, but the increased immersion also interfered with the players engagement. Aylett and colleagues [1] found that users enjoyed interaction using the dance pad and the WiiMote. However, they also realized that the interaction hampered the users activities and demanded considerable effort and concentration. To provide immersive interaction, we employ full body gestures and a swipe menu in our interactive storytelling scenario. We further apply a user-centerd design process to create an intuitive gesture set. The details of our approach are described in the following section. 


\section{Full Body Interaction in Traveller}

Traveller aims to provide intercultural training for 18-25 year olds. The users learn by participating actively in the narrative and interacting with virtual characters simulated by FAtiMA [2] and representing different synthetic cultures defined in Hofstedes dimensions [4]. The users start a journey through three different countries and in each country, they have to interact with locals in so-called critical incidents (CIs) to progress. The appropriate interaction is dependent on the agents synthetic culture.

By default, actions in Traveller are taken by performing a related full body gesture. Figure 1 on the left-hand side depicts a user performing an informal greeting represented by a waving gesture. An iterative user-centred design approach for user-defined gestures was used with Traveller to create intuitive gestures. More details on this can be found in [7]. For integrating the gestures in our application we use the full body interaction framework (FUBI) of which an earlier version was presented in [8]. To assist the users in performing the gestures we label the possible actions and display symbols that visualise how the gestures for these actions should be performed. In Figure 1, two such symbols can be seen in the left-hand image in cyan color.
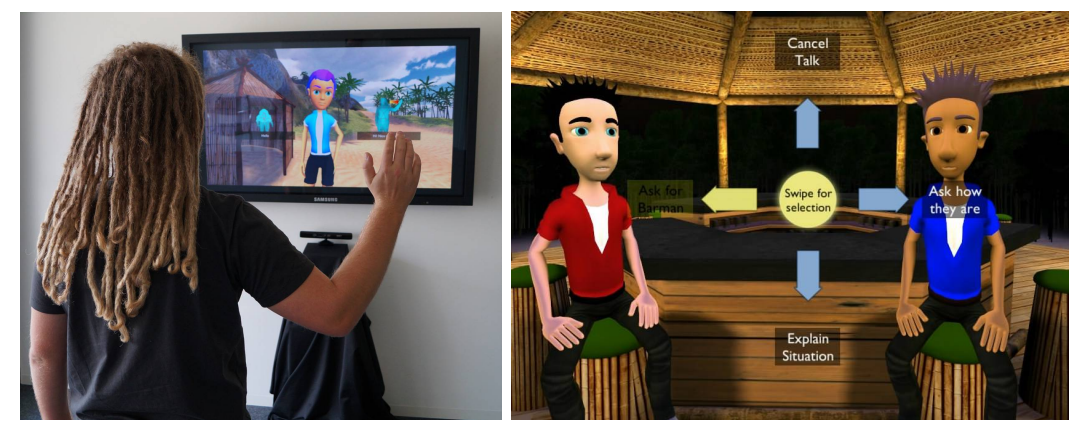

Fig. 1. Left image: User performing a greeting gesture. Right image: The swipe menu.

At certain points, we further integrate a graphical menu that includes additional dialogue options as shown in Figure 1 on the right-hand side. Within the menu, interaction options are arranged around a middle circle. For selecting an option, users first have to stretch out their hand to the front, wait until the menu gets activated, and then perform a swiping gesture in the direction of the option they would like to select. This enables us to have as many and as complex actions as we want without worrying about how all of them could be represented by unambiguous gestures. However, the two interaction types are similar enough to provide a fluent user experience.

In a single walk through the complete story, an average user selects about 50 different actions (40 by gestures and 10 by swipes) and further has to perform 36 swipes for selecting "continue". 


\section{Preliminary Evaluation}

In a first evaluation study, we had two groups of participants: one interacting with the actual full body interaction interface, and the other one interacting with a traditional mouse interface. In the latter case, participants selected the in-game actions by clicking on them with a mouse, however, we only displayed the action labels, but omitted the gesture symbols.

The full body interaction group consisted of twelve participants (two females, average age 24.3), all with German cultural background and right-handed. Regarding recognition, we counted true positives $(=T P)$, false positives $(=F P)$, false negatives $(=F N)$, and gestures obviously wrongly performed by the participants (user errors $=U E$ ). We further calculated precision, accuracy, recall and user error-rate. The results are shown in Table 1. A Pearson's chi-square test further indicated significantly less recognition errors $(F P+F N)$ for the gestures in comparison to the swipes, $\chi^{2}(1)=28.48, p<0.001$.

Table 1. Accuracy measures for the recognition of our full body gestures and swipes

\begin{tabular}{|c|c|c|c|c|c|c|c|c|}
\hline & TP & FP & FN & UE & Precision & Accuracy & Recall & User Error-Rate \\
\hline Gestures & 267 & 0 & 14 & 5 & $100,00 \%$ & $95,02 \%$ & $95,02 \%$ & $1,75 \%$ \\
\hline Swipes & 241 & 24 & 35 & 5 & $90,94 \%$ & $80,33 \%$ & $87,32 \%$ & $1,64 \%$ \\
\hline Overall & 508 & 24 & 49 & 10 & $95,49 \%$ & $87,44 \%$ & $91,20 \%$ & $1,69 \%$ \\
\hline
\end{tabular}

In the mouse interaction group, we had ten participants (two females, average age 24.6), all with German cultural background, one left-handed. We measured the time it took the participants to go through the evaluated part of the story and the number of actions they selected. With mouse interaction, they needed 5:507:42 minutes (AVG 6:30 minutes, SD 0:37 minutes) and selected 25-39 actions (AVG 31.4, SD 3.69), while they spent significantly more time with the gesturebased interaction $(t(11.31)=2.27, p<0.05, r=0.56)$ with 5:06-22:28 minutes (AVG 10:16 minutes, SD 5:43 minutes) and they selected 26-40 actions (AVG 32.4 , SD 4.58). Questionnaires revealed a tendency for higher usability $(t(20)=$ $2.00, p=0.059, r=0.41)$ and higher flow $(t(16.40)=1.98, p=0.065, r=0.44)$ with the mouse in comparison to the Kinect, similar to Dow and colleagues [3]. However, in our case, this effect was less pronounced. Furthermore, we measured a significantly higher positive affect $(t(20)=2.12, p<0.05, r=0.43)$ with the Kinect in comparison to the mouse. Apparently, participants enjoyed playing with the Kinect more than with the mouse although the interaction might have been slightly more difficult and sometimes distracted them from the application.

\section{Conclusion and Future Work}

We presented a gameplay approach incorporating full body user interaction with virtual characters, which was developed for the intercultural training scenario 
Traveller. We implemented two interaction types: symbolic full body gestures and a circular menu with freehand swiping gestures. Both types received high recognition rates, however, only the gesture interaction reached our goal of $90 \%$ recognition accuracy, while the swipe interaction stayed slightly below it. In comparison with the mouse interface, participants spend significantly more time in the game when using full body interaction. However, the novel interaction did not necessarily slow down the participants, as the fastest participants actually were using this interaction modality. Furthermore, our results indicate higher usability and flow with the mouse, but higher positive affect with the Kinect. While full body interaction might be more complicated to use, it still has the potential to increase the enjoyment in interaction.

Although we did not receive significant differences regarding immersion or spatial presence, the full body interaction might get higher scores when using it in a virtual reality setup. In addition, we plan to include further methods to help the user during the interaction such as automatic feedback why a gesture has not been recognised, and step-wise instructions for more complex gestures.

Acknowledgments. This work was partially funded by the European Commission within FP7 under grant agreement eCute (FP7-ICT-257666).

\section{References}

1. Aylett, R., Vannini, N., Andre, E., Paiva, A., Enz, S., Hall, L.: But that was in another country: Agents and intercultural empathy. In: Proc. AAMAS 2009, pp. 329-336. IFAAMAS (2009)

2. Dias, J., Mascarenhas, S., Paiva, A.: Fatima modular: Towards an agent architecture with a generic appraisal framework. In: Proc. Workshop on Standards for Emotion Modeling (2011)

3. Dow, S., Mehta, M., Harmon, E., MacIntyre, B., Mateas, M.: Presence and engagement in an interactive drama. In: Proc. CHI 2007, pp. 1475-1484. ACM, New York (2007)

4. Hofstede, G.J.: Role playing with synthetic cultures: the evasive rules of the game. Experimental Interactive Learning in Industrial Management: New approaches to Learning, Studying and Teaching p. 49 (2005)

5. Janowski, K., Kistler, F., André, E.: Gestures or speech? comparing modality selection for different interaction tasks in a virtual environment. In: Proc. Tilburg Gesture Research Meeting 2013 (2013), http://tiger.uvt.nl

6. Kadobayashi, R., Nishimoto, K., Mase, K.: Design and evaluation of gesture interface of an immersive walk-through application for exploring cyberspace. In: Proc. Automatic Face and Gesture Recognition, pp. 534-539 (1998)

7. Kistler, F., André, E.: User-defined body gestures for an interactive storytelling scenario. In: Kotzé, P., Marsden, G., Lindgaard, G., Wesson, J., Winckler, M. (eds.) INTERACT 2013, Part II. LNCS, vol. 8118, pp. 264-281. Springer, Heidelberg (2013)

8. Kistler, F., Endrass, B., Damian, I., Dang, C., André, E.: Natural interaction with culturally adaptive virtual characters. Journal on Multimodal User Interfaces 6, 39-47 (2012); 10.1007/s12193-011-0087-z

9. Raybourn, E.M., Deagle, M.E., Mendini, K., Heneghan, J.: Adaptive thinking \& leadership simulation game training for special forces officers. In: Proc. I/ITSEC 2005. NTSA (2005) 